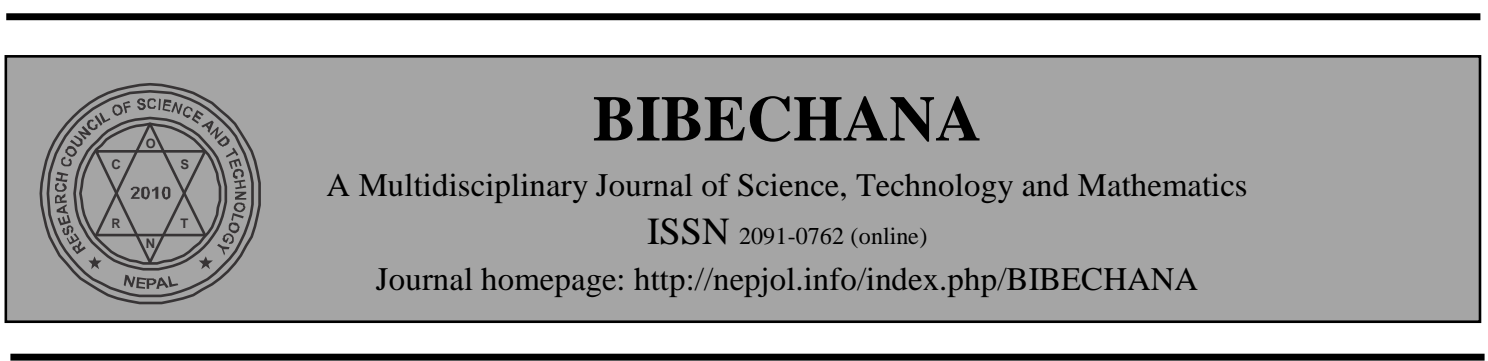

\title{
Structural study of liquid Sn-Tl alloys
}

\author{
R.P. Koirala ${ }^{1,2}$, I.S. Jha ${ }^{2}$, B.P. Singh ${ }^{1}$, D. Adhikari ${ }^{2 *}$ \\ ${ }^{1}$ University Department of Physics, T.M. Bhagalpur University, Bhagalpur, India \\ ${ }^{2}$ Department of Physics, M.M.A.M. Campus Biratnagar, Tribhuvan University, Nepal \\ *Corresponding author: E-mail: adksbdev@yahoo.com
}

Accepted for publication: February 06, 2014

\begin{abstract}
The concentration fluctuations in long wavelength limit, $\mathrm{S}_{\mathrm{cc}}(0)$ and the Warren-Cowley short range order parameter $\left(\alpha_{1}\right)$ are important microscopic functions which provide valuable information about the local arrangements of the constituent atoms in the alloy melts. In order to investigate the nature of atomic ordering in $\mathrm{Sn}$ - $\mathrm{Tl}$ alloy in the molten state at $723 \mathrm{~K}$, we have computed these functions using theoretical models. The models satisfactorily explain the small asymmetry observed in the experimental data of free energy of mixing of the alloy. The comparative study further reveals that the energy interaction parameters are temperature dependent and the Sn-Tl alloy is weakly interacting segregating system.
\end{abstract}

(c) 2014 RCOST: All rights reserved

Keywords: Free energy of mixing; Concentration fluctuation; Short range order; Segregation.

\section{Introduction}

Tin and thallium are two members among seven orphans, or metals that belong to a smaller family of elements. Tin is well known metallic element which can be alloyed with many metals. Thallium is less common metal. No large prospective applications for thallium have so far been reported. The unalloyed pure thallium is unsuitable for direct use, because of its unfavourable mechanical properties and marked tendency to oxidize. The isotope ${ }^{204} \mathrm{Tl}$ is radioactive $\left(\beta\right.$ - emmiter, $\mathrm{T}_{1 / 2}=3.5$ years $)$ while the isotope ${ }^{205} \mathrm{Tl}$ has most constant atomic vibrations (suitable for use in atomic clocks). Thallium readily forms alloys with most other metals. It forms simple eutectic alloy with tin. Thallium, like tin, lead, cadmium etc., possesses good wear resistance behaviour when used in bearings for steel shafts. As addition of thallium improves the deformation resistance, breaking strength, and hardness of lead and its alloys, metallic alloys with thallium are frequently recommended for bearings [1].

The alloys of tin with thallium are drawing attention of some authors for the past several years [2-6]. The activities of the metals in the Sn-Tl alloy at 723K show slight deviation from the Raoultian behaviour along with a small asymmetry in the free energy of mixing [6]. The interatomic interactions in the alloy determine the nature of the liquid alloy. This has aroused our interest to undertake a theoretical assessment of the structural Sn-Tl system.

As most of the commercial solid alloys derived from their corresponding liquid phase, it is important to have knowledge about the alloying behaviour of the alloys in the liquid state so that the nature of solid alloys can be understood properly. Metallic alloys in the liquid phase lack long range atomic order 
R. P. Koirala et al. / BIBECHANA 11(1) (2014) 46-52: (Online Publication: March, 2014) p.47

and they are considered as disordered systems. The physics and chemistry of such systems are normally complex phenomena. The energetics of formation in such disordered systems may result in a variety of atomic arrangements [7]. On mixing the metals in the molten state, the atoms of the constituent metals may prefer to remain self coordinated (like atoms pairing) or hetero-coordinated (unlike atoms pairing). The alloys having structural arrangement of the former type are said to be segregating and those with latter type are termed as ordering. The segregating or ordering nature of an alloy has direct influence on its thermodynamic, transport and surface properties. The nature of atomic ordering in an alloy can be comprehended from knowledge of the microscopic functions namely the concentration fluctuations in long wavelength limit, $S_{\mathrm{cc}}(0)$ and the Warren-Cowley short range order parameter, $\alpha_{1}$. The function $S_{\mathrm{cc}}(0)$ in particular is useful in ascertaining the segregation in binary liquid alloys. Moreover it can be helpful to gain insight into the stability for the binary metallic solutions. The function $\alpha_{1}$ gives the degree of ordering or segregation in the binary liquid alloy.

In the present work we intend to investigate the structural arrangement in the Sn- $\mathrm{Tl}$ alloy at $723 \mathrm{~K}$ by computing $\mathrm{S}_{\mathrm{cc}}(0)$ and $\alpha_{1}$ using two statistical mechanical methods namely the quasi-lattice theory (QLT) for weakly interacting system [8-9] and the quasi-chemical approximation (QCA) [10-11]. For the computation of $S_{\mathrm{cc}}(0)$, we take into consideration the free energy of mixing of the alloy in both models.

Necessary expressions for the theoretical analysis are presented in the next section. Section 3 deals with numerical results and discussion while conclusions are provided in section 4.

\section{Theoretical basis}

Basic expressions of the statistical models we employ in current work are presented in the following subsections.

\subsection{Quasi-lattice theory for weakly interacting alloys}

We consider statistical model of complex formation on the basis of quasi-lattice theory for weakly interacting systems [8-9]. In this approach, an interaction between the constituents of the alloys is supposed to exist in the solution due to which chemical complexes, $A_{\mu} B_{v}$ (where $\mu$ and $v$ are small integers) are formed. These complexes are given various names such as 'pseudomolecules', 'associations', 'clusters' etc. Along with these associations of unlike atoms there are also self associations between like atoms in the solution. Thus a binary alloy can be considered to be a ternary mixture of A atoms, $\mathrm{B}$ atoms and a number of complexes, $\mathrm{A}_{\mu} \mathrm{B}_{v}$. Bhatia and Singh developed compound formation model assuming the energy of an $\mathrm{AA}, \mathrm{BB}$, or $\mathrm{AB}$ bond depends on whether that bond is part of the complex or not, i.e. the energy of a given nearest bond is different if it belongs to the complex than if it does not. In the $\mathrm{Sn}-\mathrm{Tl}$ alloy at $723 \mathrm{~K}$, the observed value of $\mathrm{S}_{\mathrm{cc}}(0)$, calculated directly from observed activity data [6], shows maximum deviation from the ideal value at $\mathrm{c}_{\mathrm{Sn}}=0.6$. Thus we assume the existence of the complexes $\mathrm{Sn}_{3} \mathrm{Tl}_{2}$ in the molten state in order to understand the mixing behaviour of the $\mathrm{Sn}$-Tl alloys in the framework of quasi-lattice model for weakly interacting alloys. In this model expressions for the free energy of mixing, $\mathrm{G}_{\mathrm{M}}$; and for the microscopic functions $\mathrm{S}_{\mathrm{cc}}(0)$ and $\alpha_{1}$ can be derived by constructing grand partition function for a cluster of two lattice sites in a binary metallic solution and solving it (A detail of the formulation is available in the Ref. [8] ):

Considering Nc mole of Sn atoms and N(1-c) mole of $\mathrm{Tl}$ atoms mixed in molten state of Sn-Tl alloy, expressions have been derived which are given as follows:

\section{(i) Free energy of mixing}

The free energy of mixing, $G_{M}$ of a binary liquid solution at temperature $T$ is expressed as [8]: 
R. P. Koirala et al. / BIBECHANA 11(1) (2014) 46-52: (Online Publication: March, 2014) p.48

$$
\mathrm{G}_{\mathrm{M}}=\mathrm{Nk}_{\mathrm{B}} \mathrm{T}[\mathrm{c} \ln \mathrm{c}+(1-\mathrm{c}) \ln (1-\mathrm{c})]+\mathrm{G}_{\mathrm{M}}^{\mathrm{XS}}
$$

where $k_{B}$ is Boltzmann constant, $c$ is the concentration of A-component of the alloy. $G_{M}^{X S}$ is the excess free energy of mixing. In QLT, $\mathrm{G}_{\mathrm{M}}^{\mathrm{XS}}$ is given by:

$$
\mathrm{G}_{\mathrm{M}}^{\mathrm{XS}}=\mathrm{Nk}_{\mathrm{B}} \mathrm{T}\left[\mathrm{c}(1-\mathrm{c}) \frac{\omega}{\mathrm{k}_{\mathrm{B}} \mathrm{T}}+\phi_{\mathrm{AB}} \frac{\Delta \omega_{\mathrm{AB}}}{\mathrm{k}_{\mathrm{B}} \mathrm{T}}+\phi_{\mathrm{AA}} \frac{\Delta \omega_{\mathrm{AA}}}{\mathrm{k}_{\mathrm{B}} \mathrm{T}}+\phi_{\mathrm{BB}} \frac{\Delta \omega_{\mathrm{BB}}}{\mathrm{k}_{\mathrm{B}} \mathrm{T}}\right]
$$

Here $\omega$ 's are the ordering energies and $\phi_{\mathrm{ij}}(\mathrm{i}, \mathrm{j}=\mathrm{A}, \mathrm{B})$ are the simple polynomials in c depending on the values of $\mu$ and $\nu$. With $\mu=3$ and $\nu=2$, the values of the $\phi_{\mathrm{ij}}{ }^{\prime}$ s are given as [8]:

$$
\begin{aligned}
& \phi_{\mathrm{AB}}=\frac{13}{420} \mathrm{c}+\frac{2}{3} c^{3}-\frac{3}{2} c^{4}+\frac{3}{5} c^{5}+\frac{2}{3} c^{6}-\frac{5}{7} c^{7}+\frac{1}{4} c^{8} \\
& \phi_{\mathrm{AA}}=-\frac{53}{840} \mathrm{c}+\frac{2}{3} c^{3}-\frac{5}{4} c^{4}+\frac{6}{5} c^{5}-c^{6}+\frac{4}{7} c^{7}-\frac{1}{8} c^{8} \\
& \phi_{\mathrm{BB}}=\frac{23}{280} \mathrm{c}-\frac{1}{2} c^{4}+\frac{2}{5} c^{5}+\frac{1}{7} c^{7}-\frac{1}{8} c^{8}
\end{aligned}
$$

\section{(ii) Concentration fluctuations in the long wavelength limit, $S_{\mathrm{cc}}(\mathbf{0})$ :}

For the weakly interacting compound forming alloys, the QLT expression for $S_{c c}(0)$ is expressed as [8]:

$$
\mathrm{S}_{\mathrm{cc}}(0)=\frac{\mathrm{c}(1-\mathrm{c})}{1+\mathrm{c}(1-\mathrm{c})\left[-2 \frac{\omega}{\mathrm{k}_{\mathrm{B}} \mathrm{T}}+\frac{\Delta \omega_{\mathrm{AB}}}{\mathrm{k}_{\mathrm{B}} \mathrm{T}} \phi_{\mathrm{AB}}^{\prime \prime}+\frac{\Delta \omega_{\mathrm{AA}}}{\mathrm{k}_{\mathrm{B}} \mathrm{T}} \phi_{\mathrm{AA}}^{\prime \prime}+\frac{\Delta \omega_{\mathrm{BB}}}{\mathrm{k}_{\mathrm{B}} \mathrm{T}} \phi_{\mathrm{BB}}^{\prime \prime}\right]}
$$

where, $\phi_{\mathrm{ij}}^{\prime \prime}=\frac{\partial^{2} \phi_{\mathrm{ij}}}{\partial \mathrm{c}^{2}},(\mathrm{i}, \mathrm{j}=\mathrm{A}, \mathrm{B})$ with $\mu=3$ and $v=2$, are given by

$$
\begin{aligned}
& \phi_{\mathrm{AB}}^{\prime \prime}=4 c-18 c^{2}+12 c^{3}+20 c^{4}-30 c^{5}+14 c^{6} \\
& \phi_{\mathrm{AA}}^{\prime \prime}=4 c-15 c^{2}+24 c^{3}-30 c^{4}+24 c^{5}-7 c^{6} \\
& \phi_{\mathrm{BB}}^{\prime \prime}=-6 c^{2}+8 c^{3}+6 c^{5}-7 c^{6}
\end{aligned}
$$

(ii) Short range order parameter, Short range order parameter, $\alpha_{1}$ :

In QLT, the short range order parameter, $\alpha_{1}$ is given by [8]:

$$
\alpha_{1}=\frac{\beta-1}{\beta+1}
$$

where $\beta=\left[1+4 c(1-c)\left(\eta^{2}-1\right)\right]^{1 / 2}$

with $\quad \eta^{2}=\exp \left(\frac{2 \omega}{\mathrm{zk}_{\mathrm{B}} \mathrm{T}}\right) \cdot \exp \left(\frac{2 \mathrm{P}_{\mathrm{AB}} \Delta \varepsilon_{\mathrm{AB}}-\mathrm{P}_{\mathrm{AA}} \Delta \varepsilon_{\mathrm{AA}}-\mathrm{P}_{\mathrm{BB}} \Delta \varepsilon_{\mathrm{BB}}}{\mathrm{k}_{\mathrm{B}} \mathrm{T}}\right)$

Here, $\Delta \varepsilon_{\mathrm{ij}}(\mathrm{i}, \mathrm{j}=\mathrm{A}, \mathrm{B})$ represents the bond energy $\left(\Delta \varepsilon_{\mathrm{ij}}=\frac{\Delta \omega_{\mathrm{ij}}}{\mathrm{z}}, \mathrm{z}=\right.$ coordination number $)$ and $\mathrm{P}_{\mathrm{ij}}$ $(i, j=A, B)$ represents the probability that the bond in the cluster is part of the complex, given by 
R. P. Koirala et al. / BIBECHANA 11(1) (2014) 46-52: (Online Publication: March, 2014) p.49

$$
\begin{array}{ll}
\mathrm{P}_{\mathrm{AB}}=c^{\mu-1}(1-c)^{v-1}\left[2-c^{\mu-1}(1-c)^{v-1}\right] & \\
\mathrm{P}_{\mathrm{AA}}=c^{\mu-2}(1-c)^{v}\left[2-c^{\mu-2}(1-c)^{v}\right] & ; \mu \geq 2 \\
\mathrm{P}_{\mathrm{BB}}=c^{\mu}(1-c)^{v-2}\left[2-c^{\mu}(1-c)^{v-2}\right] & ; v \geq 2
\end{array}
$$

Assuming weak interaction in compound forming alloys where $\frac{\omega}{\mathrm{zk}_{\mathrm{B}} \mathrm{T}} \ll<1$ and $\frac{\Delta \varepsilon_{\mathrm{ij}}}{\mathrm{k}_{\mathrm{B}} \mathrm{T}} \ll<1$ (i.e. $\left.\frac{\Delta \omega_{\mathrm{ij}}}{\mathrm{zk}_{\mathrm{B}} \mathrm{T}} \ll<1\right)$, we have

$$
\eta^{2}=1+\frac{1}{\mathrm{zk}_{\mathrm{B}} \mathrm{T}}\left(2 \omega+2 \mathrm{P}_{\mathrm{AB}} \Delta \omega_{\mathrm{AB}}-\mathrm{P}_{\mathrm{AA}} \Delta \omega_{\mathrm{AA}}-\mathrm{P}_{\mathrm{BB}} \Delta \omega_{\mathrm{BB}}\right)
$$

And

$$
\beta=1+\frac{2 \mathrm{c}(1-\mathrm{c})}{\mathrm{zk}_{\mathrm{B}} \mathrm{T}}\left(2 \omega+2 \mathrm{P}_{\mathrm{AB}} \Delta \omega_{\mathrm{AB}}-\mathrm{P}_{\mathrm{AA}} \Delta \omega_{\mathrm{AA}}-\mathrm{P}_{\mathrm{BB}} \Delta \omega_{\mathrm{BB}}\right)
$$

The short range order parameter can then be obtained from the expression

$$
\alpha_{1}=\frac{x}{1+x}
$$

where $\quad \mathrm{x}=\frac{\mathrm{c}(1-\mathrm{c})}{\mathrm{zk}_{\mathrm{B}} \mathrm{T}}\left(2 \omega+2 \mathrm{P}_{\mathrm{AB}} \Delta \omega_{\mathrm{AB}}-\mathrm{P}_{\mathrm{AA}} \Delta \omega_{\mathrm{AA}}-\mathrm{P}_{\mathrm{BB}} \Delta \omega_{\mathrm{BB}}\right)$

\subsection{Quasi-chemical approximation (QCA)}

QCA for binary liquid alloys assumes the homo-coordination of like atoms at equivalent sites that results in the formation of self-associates of types $\mu \mathrm{A}$ and $v \mathrm{~B}$. In this approach the ratio of self associates $\mathrm{n}=v / \mu, \nu \neq \mu$ and order energy parameter $\mathrm{W}$ are taken as the fitting parameters to study the thermodynamic and structural properties of a liquid alloy.

\section{(i) Free energy of mixing:}

The QCA expression for the free energy of mixing, $\mathrm{G}_{\mathrm{M}}$ for the binary liquid A-B alloy is given as [1011]:

$$
\mathrm{G}_{\mathrm{M}}=\mathrm{RT}[\mathrm{c} \ln \phi+(1-\mathrm{c}) \ln (1-\phi)]+\phi(1-\phi)(\mathrm{c}+\mathrm{n}-\mathrm{nc}) \mathrm{W}
$$

where, $\mathrm{R}$ is universal gas constant. The auxiliary function $\phi$ is defined by

$$
\phi=\frac{\mathrm{c}}{\mathrm{c}+\mathrm{n}-\mathrm{nc}}
$$

\section{(ii) Concentration fluctuations in the long wavelength limit, $S_{\mathrm{cc}}(\mathbf{0})$ :}

The microscopic structure, concentration fluctuation in long wavelength limit, $\mathrm{S}_{\mathrm{cc}}(0)$ is obtained from the second composition derivative of the free energy of mixing:

$$
\mathrm{S}_{\mathrm{cc}}(0)=\mathrm{RT}\left(\partial^{2} \mathrm{G}_{\mathrm{M}} / \partial \mathrm{c}^{2}\right)_{\mathrm{T}, \mathrm{P}, \mathrm{N}}^{-1}
$$

Using Eqs. (14) and (16), we get

$$
\begin{gathered}
\mathrm{S}_{\mathrm{cc}}(0)=\frac{\mathrm{c}(1-\mathrm{c})}{1-\mathrm{c}(1-\mathrm{c}) \mathrm{f}(\mathrm{n}, \mathrm{W})} \\
\text { where } \mathrm{f}(\mathrm{n}, \mathrm{W})=\frac{2 \mathrm{n}^{2}(\mathrm{~W} / \mathrm{RT})-(\mathrm{n}-1)^{2}(\mathrm{c}+\mathrm{n}-\mathrm{nc})}{(\mathrm{c}+\mathrm{n}-\mathrm{nc})^{3}}
\end{gathered}
$$


R. P. Koirala et al. / BIBECHANA 11(1) (2014) 46-52: (Online Publication: March, 2014) p.50

The value of $S_{c c}(0)$ indicates the nature of chemical ordering in the alloy. The values of $S_{c c}(0)$ computed from the following relation are regarded as the experimental $\mathrm{S}_{\mathrm{cc}}(0)[10]$ :

$$
\mathrm{S}_{\mathrm{cc}}(0)=\frac{(1-\mathrm{c}) \mathrm{a}_{\mathrm{A}}}{\frac{\partial \mathrm{a}_{\mathrm{A}}}{\partial \mathrm{c}}}=\frac{c \mathrm{a}_{\mathrm{B}}}{\frac{\partial \mathrm{a}_{\mathrm{B}}}{\partial(1-\mathrm{c})}}
$$

(ii) Short range order parameter, Short range order parameter, $\alpha_{1}$ :

Warren-Cowley short-range parameter, $\alpha_{1}$ which represents the degree of ordering in the melt, can be estimated from the knowledge of $S_{c c}(0)$ using the following expression [10-13]:

$$
\alpha_{1}=\frac{\mathrm{S}-1}{\mathrm{~S}(\mathrm{z}-1)+1}, \quad \text { with } \quad \mathrm{S}=\frac{\mathrm{S}_{\mathrm{cc}}(0)}{\mathrm{c}(1-\mathrm{c})}
$$

where $\mathrm{z}$ is the coordination number.

\section{Results and Discussion}

In the present work we have theoretically studied structural behaviour in the liquid Sn-Tl alloys at $723 \mathrm{~K}$ by comparative method. For the computation of the microscopic functions from QLT, interatomic interaction energy parameters $\omega$ and $\Delta \omega_{\mathrm{ij}}$ 's are required while in QCA, the ratio of self associates $\mathrm{n}$ and order energy parameter $\mathrm{W}$ are needed. We have estimated the model fit parameters for the alloy using the experimental data of integral free energy of mixing [6], from Eqs. (1) and (2) in QLT and from Eq. (14) in QCA.

The best fit values of energy parameters for the computation of free energy of mixing of the alloy are found to be:

$$
\begin{aligned}
\text { QLT: } & \frac{\omega}{\mathrm{k}_{\mathrm{B}} \mathrm{T}} & =+0.69 ; \quad \frac{\Delta \omega_{\mathrm{AB}}}{\mathrm{k}_{\mathrm{B}} \mathrm{T}}=+1.38 ; & \frac{\Delta \omega_{\mathrm{AA}}}{\mathrm{k}_{\mathrm{B}} \mathrm{T}} \approx 0 ; \quad \frac{\Delta \omega_{\mathrm{BB}}}{\mathrm{k}_{\mathrm{B}} \mathrm{T}}=-0.85 \\
\text { QCA: } & \mathrm{n} & =1.23 ; \quad \frac{\mathrm{W}}{\mathrm{RT}}=+0.70 &
\end{aligned}
$$

Obviously if $\omega<0$, pairing of unlike atoms is preferred over pairing of like atoms. On the other hand, if $\omega>0$, there is a tendency of like atom pairing (self-associations). $\omega=0$ indicates random mixing of component atoms so that the mixture is perfectly disordered. We have found that $\omega>0$ for the Sn-Tl alloy at $723 \mathrm{~K}$. This shows that there is a tendency to form homo-pairs in the alloy. Using the estimated parameters, we have worked out the theoretical values of excess free energy of mixing and integral free energy of mixing from both models over the whole range of concentration. The excess free energy of mixing has been found positive at all concentrations with maximum value at $1.16 \mathrm{kJmol}^{-1}$ (QLT) and $1.13 \mathrm{kJmol}^{-1}$ (QCA) at the equi-atomic concentration. This is an indication of segregating tendency in the $\mathrm{Sn}-\mathrm{Tl}$ alloy. The theoretical results for the integral free energy of mixing are found in good agreement with the experimental result [6] as depicted in Fig. 1.

The nature and degree of local ordering of atoms in the nearest cells in a binary liquid alloy can be effectively discussed in terms of the microscopic constructs $S_{\mathrm{cc}}(0)$ and $\alpha_{1}$. Here we have computed the concentration fluctuation in the long wavelength limit, $\mathrm{S}_{\mathrm{cc}}(0)$ as function of concentration for the $\mathrm{Sn}-\mathrm{Tl}$ alloy from Eq. (4) of QLT and Eq. (17) of QCA. In order to maintain the consistency we have used the same parameters which were used in the computation of free energy of mixing. The experimental $\mathrm{S}_{\mathrm{cc}}(0)$ values are obtained directly from the observed activity data [6] using Eq. (19). The computed values of $\mathrm{S}_{\mathrm{cc}}(0)$ are plotted against concentration of Tl-component in Fig. 2 along with the observed and ideal values. The $S_{\mathrm{cc}}(0)$ calculated from both approaches are found greater than the ideal values throughout the entire concentration range. This indicates that there is segregating nature in the $\mathrm{Sn}-\mathrm{Tl}$ alloy. 
To gain a deeper understanding into the microscopic structure regarding the degree of atomic ordering in the liquid Sn-Tl alloy, we have next calculated the Warren-Cowley short range ordering parameter, $\alpha_{1}$ from Eq. (12) for QLT and from Eq. (20) for QCA. It is observed that $\alpha_{1}$ is positive at all concentrations

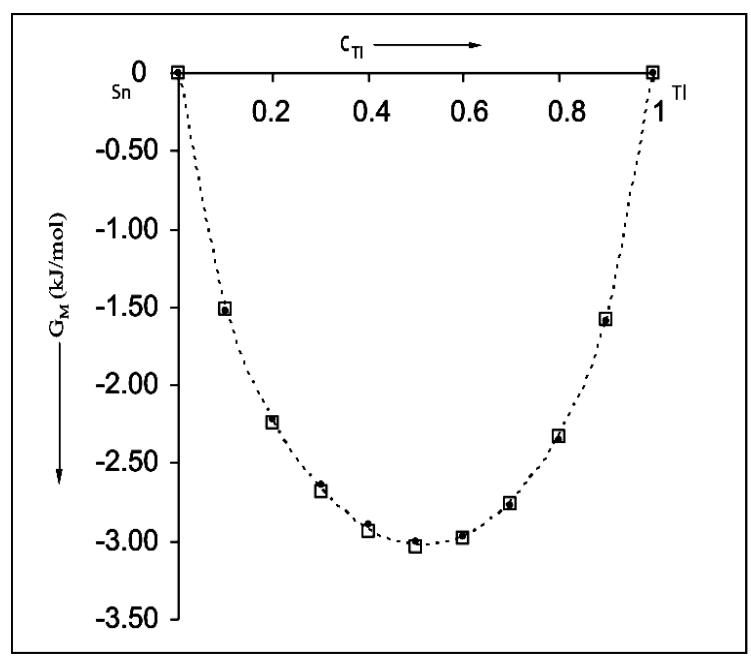

Figure 1: Free energy of mixing of Sn-Tl alloy at $723 \mathrm{~K}$ versus concentration of Tl-component: Thick dots - QLT Squares - QCA Broken curve - Experimental [6].

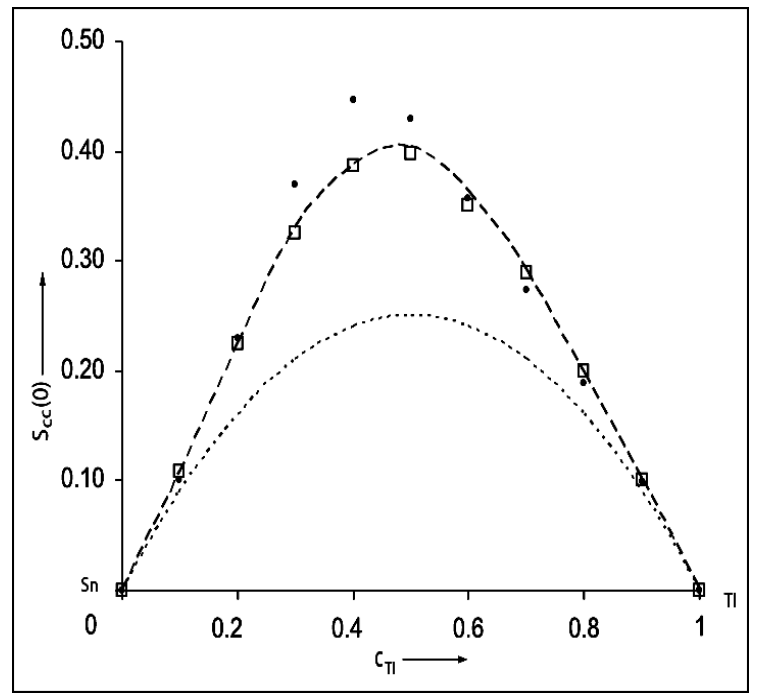

Figure 2: Concentration fluctuations in long wavelength limit of $\mathrm{Sn}-\mathrm{Tl}$ alloy at $723 \mathrm{~K}$ versus concentration of Tl-component:

Thick dots - QLT

Squares - QCA

Thick broken line - Experimental

Thin broken line - Ideal.

(Fig. 3). The positive values of $\alpha_{1}$ confirms the preference of self association over hetero-coordination between the unlike atoms in the $\mathrm{Sn}-\mathrm{Tl}$ alloy as observed from the result of $\mathrm{W}, \mathrm{S}_{\mathrm{cc}}(0)$ and the excess free energy of mixing. In this study as $\alpha_{1}<<1$, there is weak interaction among the species of the liquid Sn-Tl alloy at $723 \mathrm{~K}$. It is clear from Fig. (3) that there is larger segregation in the alloy towards Sn-rich side of the concentration. Along with the study of the microscopic structure, we have also examined the validity of two statistical methods to explain the behaviour of the $\mathrm{Sn}-\mathrm{Tl}$ alloy in the molten state. In our analysis quasi-chemical approximation is found more appropriate approach for the $\mathrm{Sn}$-Tl alloy than quasi-lattice theory in weak interaction indicating very weak complex $\mathrm{Sn}_{3} \mathrm{Tl}_{2}$ likely to exist in the alloy at $723 \mathrm{~K}$. 
R. P. Koirala et al. / BIBECHANA 11(1) (2014) 46-52: (Online Publication: March, 2014) p.52

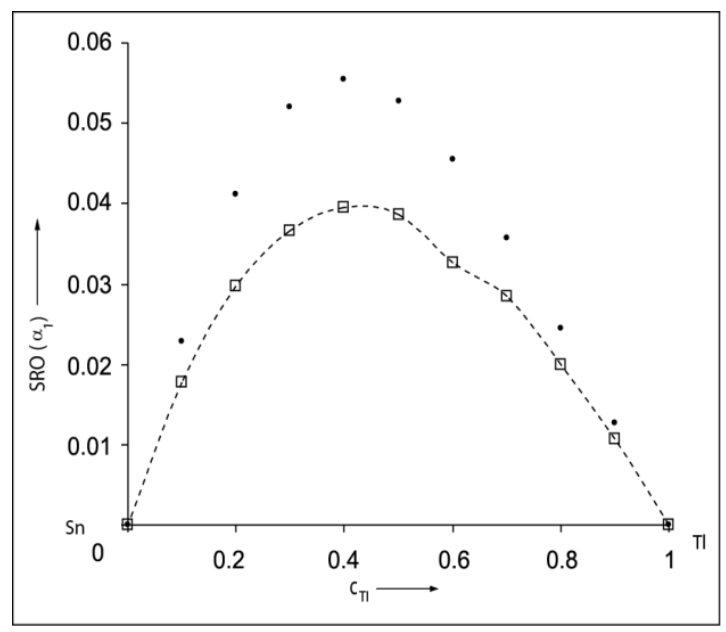

Figure 3: Warren-Cowley short range order parameter of $\mathrm{Sn}-\mathrm{Tl}$ alloy at $723 \mathrm{~K}$ versus concentration of $\mathrm{Tl}-$ component: Thick dots -QLT

Dashed line with squares - QCA.

\section{Conclusion}

We have performed a theoretical study of the free energy of mixing and the microscopic structures of Sn$\mathrm{Tl}$ alloy in the molten state using two approaches. Our theoretical analysis suggests that the alloy is a weakly interacting segregating system. Further the quasi-chemical approximation seems more appropriate than the quasi-lattice theory in explaining the thermodynamic behaviour of the $\mathrm{Sn}-\mathrm{Tl}$ alloy at $723 \mathrm{~K}$.

\section{Acknowledgments}

We are grateful to Prof. Dr. Pradeep Raj Pradhan, Post Graduate Department of Physics, M.M.A.M. Campus, Biratnagar, Tribhuvan University, Nepal for inspiring discussion and encouragement.

\section{References}

[1] Fathi Habashi (Editor), Alloys: Preparation, Properties \& Applications, John Wiley \& Sons, 2008.

[2] P.E. Berthou, R. Tougas, Metallurgical Transactions, 3 (1972) 51.

[3] E.Yu.Tonkov, I.L. Aptekar', V.K. Gartman, Russian Journal of Physical Chemistry, 44 (1970) 1490.

[4] P.E. Berthou , Journal of the Less-Common Metals, 16 (1968) 465.

[5] Ryoji Suganuma, J. Physical Society of Japan, 15 (1960) 1395.

[6] R. Hultgren, P.D. Desai, D.T. Hawkins, M. Gleiser, and K.K. Kelly, Selected Values of the Thermodynamic Properties of Binary Alloys, ASM, Metal Park, 1973.

[7] P.J. Tumidajski, A. Petric, T. Takenaka, A.D. Pelton, M.L. Saboungi, J. Phys.: Cond. Mat., 2 (1990) 209.

[8] R.N. Singh, Can. J. Physics, 65 (1987) 309.

[9] B.P. Singh, D. Adhikari, I.S. Jha, Phys. and Chem. of Liq., 50 (2012) 697.

[10] R.N. Singh, F. Sommer, Rep. Prog. Phys., 60 (1997) 57.

[11] B.C. Anusionwu, C.A. Madhu, C.E. Orji, Pramana: J. Physics, 72 (2009) 951.

[12] R. Novakovic, J. Non-Cryst. Solids, 356 (2010) 1593.

[13] B.P. Singh, D. Adhikari, I.S. Jha, B.C. Kumar, S.K. Chaudhary, S.K. Jayaswal, R.P. Koirala, BIBECHANA, 7 (2011) 1. 